\title{
Creeping towards broader clinical application of PET myocardial blood flow quantification
}

\author{
Paul C. Cremer, MD, ${ }^{a}$ Frank P. DiFilippo, PhD, ${ }^{b}$ and Manuel D. Cerqueira, MD $^{a, b}$ \\ a Department of Cardiovascular Imaging, Heart and Vascular Institute, Cleveland Clinic, \\ Cleveland, $\mathrm{OH}$ \\ b Department of Nuclear Medicine, Cleveland Clinic Foundation, Cleveland, $\mathrm{OH}$
}

Received Jun 21, 2018; accepted Jun 21, 2018

doi:10.1007/s12350-019-01651-w

\section{See related article, pp. 719-728.}

The value of an imaging test is assessed according to diagnostic accuracy, improved risk stratification, and downstream management implications. In clinical practice, the enthusiasm for incorporating measurements of absolute myocardial blood flow (MBF) with dynamic cardiac PET is based on the excellent performance within these categories. Specifically, myocardial flow reserve (MFR) has consistently provided incremental risk stratification beyond gated and relative perfusion variables. ${ }^{1,2}$ More recently, PET MBF has also been used to diagnose and predict adverse events related to coronary microvascular dysfunction. ${ }^{3,4}$ In addition, visual interpretation of both SPECT and PET uses normalized or relative perfusion images that are limited by artifacts and less accurate in the presence of balanced or severe diffuse disease. Finally, preserved MFR can exclude high-risk $\mathrm{CAD}$ and may therefore aid in appropriate patient selection for subsequent invasive testing. 5,6

In part due to the added value of MBF measurements, rest-stress myocardial perfusion PET may be a preferred, first-line test in certain clinical scenarios. ${ }^{7}$ Despite all of these favorable characteristics, and having been performed for over 30 years, the technique has been limited to research applications and is employed at a limited number of hospital based, predominately academic medical centers where it may not be included in the final report. The majority of published data are

Reprint requests: Paul C. Cremer, MD, Department of Cardiovascular Imaging, Heart and Vascular Institute, Cleveland Clinic, Cleveland, OH 44195; paul.cremer@gmail.com

J Nucl Cardiol 2019;26:735-7.

1071-3581/\$34.00

Copyright (C) 2019 American Society of Nuclear Cardiology. predominantly retrospective and from single-centers. ${ }^{6}$ As MBF assessments have become more widespread, potential factors to consider and pitfalls in data acquisition and analysis have been highlighted and are summarized in Table $1 .{ }^{8}$ Indeed, even before attempting to ascribe value to an imaging test, the implicit assumption is that the test is highly accurate with robust reproducibility. Given the number of options, factors to consider, and the potential errors that can be introduced, agreement on what to measure and normal values have not been defined.

In the current issue, Koenders et $\mathrm{al}^{9}$ address a basic and important point related to accuracy and reproducibility, myocardial creep. Within this context, myocardial creep refers to altered myocardial blood flow measurements due to changes in diaphragmatic position after vasodilator administration. Specifically, the authors defined myocardial creep as misalignment of at least one-third of the width of the left ventricle in 2 time frames including filling of the left ventricle during the first pass phase. If myocardial creep was identified, manual re-alignment of the contours was performed, and the outcome was a difference in MBF or MFR of at least $10 \%$ between corrected and uncorrected scans.

According to this definition, in this retrospective cohort study of 104 patients, approximately half demonstrated myocardial creep during the stress acquisition. Although the magnitude of motion was not quantified, the high prevalence of visually significant creep is an obstacle in applying MBF in clinical practice. As the authors demonstrate, myocardial creep is especially a concern in the RCA distribution where misalignment can lead to inappropriate counts in the inferior wall when the signal is actually from the left ventricular cavity. Their results are consistent with this observation where the mean stress MBF in the RCA distribution decreased from 4.0 to $2.7 \mathrm{~mL} / \mathrm{min} / \mathrm{g}$. 
Table 1. Technical factors affecting MBF quantitative accuracy

\section{Technical factor}

\section{Impact}

\author{
I. Scanner \\ A. Spatial resolution \\ B. Data corrections (deadtime, randoms, \\ scatter) \\ C. Detector saturation \\ D. Time-of-flight (TOF) capability \\ II. Technique
A. Infusion characteristics
B. Timing (relative to scan start)
C. CT acquisition (breath-hold, free- breathing) versus separate $\mathrm{CTs}$ )
A. Reconstruction algorithm and parameters
B. Dynamic framing
C. PET/CT registration (capability and quality of registration correction)
D. Motion correction (real-time, or post- processing)
A. Software selection
B. Motion correction (frame-by-frame)
C. MBF algorithm
D. Blood pool ROI definition \\ D. Protocol (single CT for both rest/stress \\ III. Image generation \\ IV. MBF analysis
}

Spillover/partial volume errors

Quantitative errors

Quantitative errors; reproducibility depends on infusion technique Image quality difference between scanners

Bolus vs sustained infusion, bolus peak depends on age of Rb-82 generator and IV line quality, possible double-peaks

Potential for missed bolus or inconsistency

Attenuation-related quantitative errors and artifacts

Attenuation-related quantitative errors and artifacts

Noise characteristics, convergence errors, potential bias in MBF

Timing of frames may affect MBF

Attenuation-related quantitative errors and artifacts

Attenuation-related quantitative errors and artifacts

Vendor-dependent MBF results

Availability of tools to inspect and correct (automated, manual, both, or neither?)

Differences between flow-extraction models, errors at high flow Quantitative errors
Even though Koenders et al do not provide data regarding subsequent testing and adverse events, the high incidence and magnitude of myocardial creep could certainly impact diagnostic accuracy. Therefore, routine quality control in a rest-stress MBF assessment should include inspection of time activity curves and frame-byframe inspection of dynamic data. ${ }^{10}$ In this regard, MBF analysis software must provide user-friendly tools for frame-by-frame inspection and correction of patient motion. As the authors highlight, increased signal in the RCA distribution compared to the LAD and LCX on the first pass phase of the time activity curve is compatible with differential spillover from the left ventricle because myocardial uptake has not yet occurred. Similarly, during the first pass phase, spillover from the left ventricle into the automatically drawn myocardial contour may be observed in the inferior wall.
Overall, Koenders et al and others have made a valuable contribution to the literature highlighting the importance of blood pool phase patient motion during MBF measurements. ${ }^{11,12}$ Recently, Memmott et al have shown that patient motion is more common with adenosine compared to regadenoson and hypothesized that patient motion may be most pronounced at termination of the adenosine infusion. ${ }^{11}$ In addition, Lee et al demonstrated that blood phase motion in the inferior direction resulted in mean MBF and MFR errors of 29$44 \%$ in the RCA territory, ${ }^{12}$ results consistent with the current manuscript.

Within a broader context, these papers all highlight the importance of technical considerations in measuring and reporting MBF measurements, especially as it relates to clinical reporting in different labs and the inclusion of MBF assessments within multi-center 
clinical trials. ${ }^{10}$ Accordingly, it is worth emphasizing the best-case scenario for test-retest variability and highlighting sources of methodological variation. In a singlecenter study, Kitkungvan et al demonstrated that the coefficient of variance for serial studies performed minutes apart was approximately $10 \%$ and was $20 \%$ for studies performed days apart. ${ }^{13}$ This precision of $\pm 20 \%$ reflects both methodological and biological variability, and importantly, defines probability bounds in which clinical decisions could be made with certainty.

This methodological variation of $10 \%$ represents an ideal scenario with uniform protocols and equipment performed at a single center. Putting aside the choice of radiopharmaceutical and vasodilator, which will also effect MBF measurements, ${ }^{14}$ what technical aspects will further influence this methodological variation? In general, considerations relate to the following domains: scanner parameters, radiopharmaceutical administration, methods of attenuation and motion correction, image reconstruction, and MBF analysis (Table 1). Given the equipment and software options and technical demands of the technique, professional medical societies and laboratory accreditation bodies need to provide measurement standardization and quality control guidance on how to perform absolute PET MBF measurements. The work by Koenders et al highlights just one of the important variables influencing measurements.

\section{Disclosure}

Paul C. Cremer, Frank P. DiFilippo, and Manuel D. Cerqueira have nothing to disclose.

\section{References}

1. Ziadi MC, Williams KA, Guo A, Chow BJ, Renaud J, Ruddy TD, et al. Impaired myocardial flow reserve on Rubidium- 82 positron emission tomography imaging predicts adverse outcomes in patients assessed for myocardial ischemia. J Am Coll Cardiol 2011;58:740-8.

2. Murthy VL, Naya M, Foster CRE, Hainer J, Gaber M, Di Carli G, et al. Improved cardiac risk assessment with non-invasive measures of coronary flow reserve. Circulation 2011;124:2215-24.

3. Taqueti VR, Solomon SD, Shah AM, Desai AS, Groarke JD, Osborne MT, et al. Coronary microvascular dysfunction and future risk of heart failure with preserved ejection fraction. Eur Heart $\mathbf{J}$ 2018;39:840-9.

4. Taqueti VR, Hachamovitch R, Murthy VL, Naya M, Foster CR, Hainer J, et al. Global coronary flow reserve is associated with adverse cardiovascular events independently of luminal angiographic severity and modifies the effect of early revascularization. Circulation 2015;131:19-27.

5. Gould KL, Johnson NP, Bateman TM, Beanlands RS, Bengel FM, Bober R, et al. Anatomic versus physiologic assessment of coronary artery disease: Role of coronary flow reserve, fractional flow reserve, and positron emission tomography imaging in revascularization decision making. J Am Coll Cardiol 2013;62:1639-53.

6. Naya M, Murthy VL, Taqueti VR, Foster CR, Klein J, Garber M, et al. Preserved coronary flow reserve effectively excludes highrisk coronary artery disease on angiography. J Nucl Med 2014;55:248-55.

7. Bateman TM, Dilsizian V, Beanlands RS, DePuey EG, Heller GV, Wolinsky DA, et al. American Society of Nuclear Cardiology and Society of Nuclear Medicine and Molecular Imaging joint position statement on the clinical indications for myocardial perfusion PET. J Nucl Cardiol 2016;57:1654-6.

8. Cremer PC, DiFilippo FP, Jaber WA. Moving towards a better understanding of potential pitfalls in quantitative PET myocardial blood flow. J Nucl Cardiol. 2017. https://doi.org/10.1007/s12350017-0828-9.

9. Koenders S, van Dijk J, Jager P, Ottervanger JP, Slump CH, van Dalen JA (2018) Impact of regadenoson induced myocardial creep on dynamic Rubidium-82 PET myocardial blood flow quantification. J Nucl Cardiol

10. Murthy VL, Bateman TM, Beanlands RS, Berman DS, BorgesNeto S, Chareonthaitawee P, et al. Clinical quantification of myocardial blood flow using PET: Joint position paper of the SNMMI cardiovascular council and the ASNC. J Nucl Med 2018;25:269-97.

11. Memmott MJ, Tonge CM, Saint KJ, Arumugam P (2017) Impact of pharmacological stress agent on patient motion during rubidium-82 myocardial perfusion PET/CT. J Nucl Cardiol

12. Lee BC, Moody JB, Poitrasson-Riviere A, Melvin AC, Weinberg RL, Corbett JR, et al. Blood pool and tissue phase patient motion effects of rubidium-82 PET myocardial blood flow quantification. J Nucl Cardiol. 2018. https://doi.org/10.1007/s12350-018-1256-1.

13. Kitkungvan D, Johnson NP, Roby AE, Patel MB, Kirkeeide R, Gould KL. Routine clinical quantitative rest and stress myocardial perfusion for managing coronary artery disease: Clinical relevance of test-retest variability. J Am Coll Cardiol Imaging 2017;10:56577.

14. Johnson NP, Gould KL. Regadenoson versus dipyridamole hyperemia for cardiac PET imaging. J Am Coll Cardiol Imaging 2015;8:438-47.

Publisher's Note Springer Nature remains neutral with regard to jurisdictional claims in published maps and institutional affiliations. 